\title{
FHplus Assessment (Executive Summary)
}

Im Auftrag der TIG (Technologie Impulse Gesellschaft mbH) nunmehr FFG - Österreichische Forschungsförderungsgesellschaft

Bereich 2: Kooperation Wissenschaft / Wirtschaft

Anton Geyer

September 2004

Technopolis Forschungs- und Beratungsgesellschaft $\mathrm{mbH}$

Prinz Eugen Straße 80/12

1040 Wien

Tel. 015039592 - 12

anton.geyer@technopolis-group.com

www.technopolis-group.com 


\section{Executive Summary}

Die österreichischen Fachhochschulen und FH-Studiengänge unterscheiden sich hinsichtlich der vorhandenen Erfahrungen, ihrer Kompetenzen und ihres Potenzials für Forschung und Entwicklung beträchtlich. Mit dem FHplus Programm sollen unabhängig von der jeweiligen Ausgangslage die Strukturen und Kapazitäten für anwendungsbezogene Forschung gestärkt, und längerfristige FuE Kooperationen mit der Wirtschaft und anderen FuE Partnern intensiviert werden. Dieser doppelten Zielsetzung wird durch die beiden Vorhabenskategorien ,Strukturaufbau-Vorhaben' und ,Kooperationsvorhaben' entsprochen.

\section{Projekthintergrund}

TIG Technologie Impulse Gesellschaft (nunmehr Bereich 2: Kooperation Wissenschaft / Wirtschaft der FFG - Österreichische Forschungsförderungsgesellschaft) beauftragte Technopolis Forschungs- und Beratungsgesellschaft m.b.H. und $3 \mathrm{~s}$ Unternehmensberatung GmbH mit einem Assessment der bisherigen Erfahrungen mit der Durchführung des Impulsprogramms FHplus. Ziel des Assessments war es, aus den vorliegenden Erfahrungen mit FHplus für die Vorbereitung der zweiten Ausschreibung (deren Start für Herbst 2004 geplant ist) zu lernen und gegebenenfalls Vorschläge für eine weitere Verbesserung von Ausschreibung und Programmumsetzung zu formulieren.

Das Assessment wurde in zwei Teile gegliedert. Zum einen führte $3 \mathrm{~s}$ eine online Befragung unter Akteuren des Fachhochschulsektors über ihre Einschätzung des FHplus Programms durch. Technopolis führte parallel dazu eine Analyse des bisherigen Programmmanagements durch. Grundlage dafür waren die vorhandenen Programmunterlagen und die verfügbaren Dokumente zu den eingereichten bzw. geförderten Vorhaben. Von beiden beauftragten Einrichtungen wurden zusätzlich mit den wichtigsten Akteuren und Adressaten des FHplus Programms Interviews geführt. Angesprochen wurden RepräsentantInnen der Fachhochschulen, VertreterInnen der beteiligten Ministerien, der Fachhochschulrat, der Rat für Forschung und Technologieentwicklung, sowie die Mitglieder der ExpertInnenjury, die in der ersten Ausschreibung die Projekte beurteilten bzw. zur Förderung vorschlugen.

\section{Analyse des FHplus Programmmanagements: Ergebnisse und Empfehlungen}

Die Ergebnisse der Analyse des bisherigen Managements des FHplus Programms bestätigen die Notwendigkeit und Angemessenheit des Programms in Bezug auf Konzeption und Zielsetzungen. Die erste Ausschreibung stieß auf großes Interesse. Bis auf eine Einrichtung reichten alle antragsberechtigten Erhalter Vorhaben zur Förderung im FHplus Programm ein. Im Vergleich zu anderen Programmen und Förderinstrumenten in Österreich wies die erste Ausschreibungsrunde eine sehr hohe Überzeichnung auf. Das Volumen der beantragten Bundesförderung der eingereichten 65 Vorhaben übertraf etwa um das Vierfache die zur Verfügung stehenden Finanzmittel. Insgesamt wurden von einer international besetzten Expertenjury 20 Vorhaben zur Förderung empfohlen, wobei 10,6 Mio. Euro an Bundesmitteln bewilligt wurden. 
Die hohe Überzeichnungs- und Ablehnungsrate muss jedoch im Lichte der Anforderungen, Instrumente und Beurteilungskriterien des FHplus Programms relativiert werden. Die Jurybegründungen für abgelehnte Vorhaben legen nahe, dass in zahlreichen Anträgen zu wenig darauf geachtet wurde, den für eine positive Beurteilung notwendigen struktur- und kompetenzbildenden Charakter der geplanten FuE-Vorhaben herauszuarbeiten. Es wurden zum Teil Vorhaben eingereicht, die zwar für die jeweiligen Fachhochschulen und FH-Studiengänge durchaus relevante FuE Projekte darstellten. Insgesamt entsprachen sie jedoch nicht den Zielsetzungen des FHplus Programms, weil eine längerfristige, über das Einzelprojekt hinausgehende und in eine Forschungsstrategie des Erhalters eingebettete Orientierung des Vorhabens fehlte. Um in der zweiten Runde derartige Anträge zu vermeiden, sollte in der Kommunikation mit den Fachhochschulen das zentrale Programmziel des Struktur- und Kompetenzaufbaus noch stärker betont werden.

Die Ausschreibungen des FHplus Programms richten sich an alle Disziplinen und Fachgebiete, unabhängig von der thematischen Orientierung der Fachhochschulen bzw. der FH-Studiengänge. Von Seiten einzelner Fachhochschulen wurde allerdings der technikorientierte Charakter des Programms kritisiert. Tatsächlich finden sich in den Programmdokumenten Formulierungen und Anforderungen, von denen sich technisch ausgerichtete Fachhochschulen möglicherweise eher angesprochen fühlen als andere Studiengänge. Andererseits kann argumentiert werden, dass dies eine Folge der Programmkonzeption selbst ist, da die Stärkung von Kooperationen mit der Wirtschaft ein zentrales Programmziel darstellt und technische Studiengänge eben häufig wirtschaftsnäher positioniert sind. Gleichzeitig ist aber festzuhalten, dass auch Anträge mit wirtschaftlicher bzw. sozialwissenschaftlicher Ausrichtung die Beurteilungskriterien erfüllen konnten und erfolgreich eingereicht wurden. Trotzdem ist eine neutralere Wortwahl in den Antragsunterlagen in der zweiten Ausschreibung wünschenswert.

Die Unterscheidung der beiden Vorhabenskategorien ,Strukturaufbau-Vorhaben' und ,Kooperationsvorhaben' sollte sich auch in den jeweiligen Beurteilungskriterien, bzw. in der relativen Gewichtung der einzelnen Kriteriensätze, widerspiegeln. Für beide Vorhabenskategorien sollten die Kriteriensätze unbedingt gestrafft und präzisiert werden. Die Vorhabenskategorie ,Strukturaufbau-Vorhaben' stellt ein Alleinstellungsmerkmal des FHplus Programms im Vergleich zu anderen FuEFörderprogrammen dar. Die Konzeption der Vorhabenskategorie ,Kooperationsvorhaben' ist weniger programmspezifisch. Eine noch klarere konzeptionelle Betonung des Aspekts des Kompetenzaufbaus im Design der Vorhabenskategorie (auch in Abgrenzung von anderen FuE-Fördermechanismen die Fachhochschulen zur Verfügung stehen) ist anzustreben.

Leitfaden und Einreichunterlagen für die erste Ausschreibung wiesen inhaltliche und gestalterische Defizite auf die deren Nutzerfreundlichkeit beeinträchtigte. Die Struktur und Gestaltung der Leitfäden und Einreichformulare sollte dahingehend überarbeitet werden, dass eine adäquate Darstellung der Ziele, Inhalte und Organisation der Vorhaben durch die Einreicher bestmöglich unterstützt wird. Gleichzeitig sollen sie den FachgutachterInnen und Jurymitgliedern ausreichend Orientierung für die Beurteilung der Vorhaben getrennt nach Vorhabenskategorie bieten. Ein praktischer Schritt in diese Richtung wäre eine graphisch-gestalterische 
Unterscheidung der Unterlagen für die beiden Vorhabenskategorien ,StrukturaufbauVorhaben' und ,Kooperationsvorhaben'.

Die Organisation und Durchführung des Auswahlverfahrens durch die TIG erfolgte im Einklang mit den Bestimmungen der FHplus Sonderrichtlinien in fairer, transparenter und unabhängiger Art und Weise. Trotzdem gab es nach der ersten Ausschreibung von Seiten einzelner Fachhochschulen Kritik am Auswahlprozedere. Dies kann vor allem auf die Vorgangsweise zur Auswahl von FachgutachterInnen zurückgeführt werden. Die Benennung möglicher KandidatInnen durch die EinreicherInnen war eine Quelle für beträchtliche Missverständnisse über deren Rolle und Funktion im Auswahlverfahren. In kommenden Ausschreibungen sollten die FachgutachterInnen direkt durch das Programmmanagement bestellt werden. Vorhaben eines Fachgebiets sollten durch die selben FachgutachterInnen beurteilt werden, um die Vergleichsmöglichkeiten zwischen den eingereichten Vorhaben zu verbessern.

Zusätzlich wäre zu überlegen, das Programmmanagement mit dem Vorsitz und der Moderation in der Jurysitzung zu betrauen. Damit verbunden wäre die Erweiterung der Jury um die VertreterInnen der beteiligten Ministerien. Durch diese Maßnahme wäre zweierlei sichergestellt: Erstens eine gleichrangige Rolle der beteiligten Ministerien im Auswahlverfahren, in dem sie die forschungspolitischen Interessen und Ziele als Geldgeber repräsentieren. Zweitens, die Sicherstellung einer möglichst effizienten Vorbereitung und Abwicklung des Juryverfahrens gemäß den Bestimmungen der Sonderrichtlinie und im Einklang mit den Erfordernissen des Programmmanagements.

Die Vorschriften für das FHplus Berichtswesen entsprechen den Anforderungen des finanziellen Projektmonitorings durch das Programmmanagement. Durch entsprechende Vorgaben zur Strukturierung der Jahresberichte könnte das inhaltliche Monitoring des Projektfortschritts besser in Beziehung zu den Vorhabens- und Programmzielen gesetzt werden. Insgesamt ist anzustreben, dass das Berichtswesen der geförderten Vorhaben als ein Instrument zur Unterstützung des Projektmanagements genutzt werden kann. Ein begleitendes Informationsangebot, z.B. in Form von Workshops zum Thema ,Nutzung des Berichtswesens für das Projektmanagement' wäre hilfreich.

Das Verfahren zur Vorbereitung und Genehmigung einer Ausschreibung involviert das BMBWK, das BMVIT, den Rat für Forschung und Technologieentwicklung und das Bundesministerium für Finanzen. Das komplexe Entscheidungsverfahren über die Durchführung und Dotierung einer neuen Ausschreibung, das immer wieder von Neuem durchlaufen werden muss, steht sowohl im Widerspruch zum Geist der Rahmenrichtlinien als auch zu den Erwartungen der Adressaten betreffend Programmkontinuität und Ausschreibungsrhythmus. Auch wenn durch diese Vorgangsweise möglicherweise eine höhere Mobilisierung der Adressaten erreicht wird (weil jede Ausschreibung die letzte sein könnte) beeinträchtigt eine als zu erratisch wahrgenommene Programmumsetzung die Akzeptanz und Relevanz des FHplus Programms. Gleichzeitig erschwert sie eine glaubwürdige Kommunikationsarbeit. Mehr Planungssicherheit durch eine mehrjährige Budgetzuteilung wäre daher jedenfalls anzustreben. 